[Berliner entomologische Zeitschrift XVIII. 1874.]

Ueber die Varietätenbildung unter den Schmetterlingen mit Bezug auf die darwinistische Theorie. Vortrag ${ }^{1}$ ) von Dr. Staudinger in der 4ten Sitzung der naturwissenschaftlichen Gesellschaft Isis zu Dresden am 8. Mai 1873. Nach dem Referat in den Sitzungsberichten 1873. 4-9. pag. $77-79$.

In Betreff des Begriffs der Varietät unterscheidet er mehrere Arten von Varietäten. Als erste nennt er die zufälligen Abänderungen (Aberrationen), die bei einer und derselben Art an einer und derselben Oertlichkeit auftreten. Aus dieser können sich eventuell, je nachdem sie für die Art nützlich oder angenehm sind (durch natürliche Züchtung oder geschlechtliche Zuchtwahl), allmälig neue Formen, respective Arten entwickeln. Als Beispiel hierfür zeigt er einige auffallende Aberrationen von Arctia Caja L. und eine Reihenfolge von ihm selbst auf Island gezüchteter Cidaria truncata Hufn., die so aufserordentlich abändern, dafs der englische Autor Wood aus noch weniger verschiedenen Stücken dieser Art eine Anzahl Arten aufstellte.

Die zweite Art der Varietäten findet er in den Local-Varietäten oder Rassen, die man als werdende Arten betrachten kann, wenn sie zur Zeit auch noch durch mannigfache Uebergänge mit der Stammart verbunden sind. Diese Local-Varietäten treten nie neben einander, sondern nur an getrennten Localitäten auf, unterscheiden sich aber von den sogenannten vicarirenden Arten dadurch, dafs letztere niemals Uebergänge zu einander zeigen und deshalb wohl nur in ganz verschiedenen Faunengebieten auftreten. Als Beispiele von Localvarietäten zugleich mit mannigfachen Uebergängen in einander werden vorgezeigt Zygaena occitanica Vill. aus Südfrankreich, die bei Granada in der weifs werdenden Form albicans Staud, auftritt, in Catalonien in der ganz dunklen Iberica Staud. Ferner Zygaena Rhadamanthus Esp. aus

1) Bei der leider geringen Anzahl lepidopterologischer Aufsätze, welche unsere Zeitschrift bringt, dürfte die Wiedergabe dieses kurzen Vortrags um so mehr von Interesse sein, als später an denselben weiter angeknüpft werden soll.

Die Redaction. 
Südfrankreich, die gleichfalls in Catalonien statt rother ganz schwarze Hinterflügel bekommt und als Kiesenwetteri Herr.-Sch. beschrieben wurde. Dann eine Reihenfolge von Zygaena Ephialtes L., die unter acht Artnamen beschrieben worden, von denen Dorfmeister in Steyermark durch die Zucht aus den Eiern desselben Weibchens fünf bis sechs Formen erhielt, die dort als Aberrationen auftreten, während sich in Norddeutschland und in Griechenland, als den äufsersten Verbreitungs-Bezirken dieser Art, die eine oder andere dieser steyerischen Aberrationen bereits als feste Localvarietäten herausgebildet haben. Von 'Tagfaltern zeigte er noch $\mathbf{V a}$ nessa urticae L., fast überall in Europa zu Hause, die sich auf den Inseln Corsica und Sardinien als Ichnusa Bon. herausgebildet hat, in der Polarregion als polaris Staud. Von dieser europäischen Art wird als die vicarirende des nordamarikanischen Faunengebiets $\mathbf{V a}$ nessa Milberti God. vorgezeigt.

Eine dritte Art der Variation bilden die Zeitvarietäten. Diejenigen Species, die in einem Jahre zwei oder mehrere Generationen durchmachen, erscheinen in der zweiten Generation z. B. oft ganz anders, als ihre Eltern in der ersten waren u. s. w. Die Ursache hiervon kann entweder nur in der verschiedenen Dauer des Puppenzustandes (bei der einen Form öfters mebr Monate als bei der anderen Tage) oder in der verschiedenen Temperatur, die zur Zeit der Ausbildung des Schmetterlings in der Puppe herrscht, liegen, welches letztere für das Wahrscheinlichere gehalten wird. Als Belege hierfür werden Araschnia Levana L. und Prorsa L. vorgezeigt; erstere die Frühlings-, letztere die Sommer-Generation (Varietät). Ferner Anthocharis Belemia Esp. und Glauce Hübn., wo vom Vortragenden selbst aus den Eiern eines und desselben Weibchens der Anth. Belemia nach Verlauf von etwa einem Monat die zweite Generation Glauce erzogen wurde, während einige Puppen, die liegen blieben, sich im nächsten Frühling wieder als $B e$ lemia entwickelten.

Als eine vierte Art der Varietäten kann man die FutterVarietäten bezeichnen; die verschiedene Nahrung der Raupen erzeugt zuweilen verschiedene Formen. Obwohl es eine sehr grofse Anzahl polyphager Arten giebt und man nicht selten geglaubt hat, dafs ganz verschiedenes Futter auch auf die Färbung verschieden einwirken müsse, so ist dieses doch bisher nur sicher bei zwei Arten nachgewiesen, nämlich bei Ellopia Prosapiaria L., die rothbraun aussieht, wenn deren Raupe auf der Kiefer lebt und grün als prasinaria Hübn. erscheint, wenn die Raupe auf der Fichte 
lebt. Das zweite Beispiel ist Cidaria variata Schäff. und obeliscata Hübn., von denen die Raupe der ersteren (grauen) Form auch auf der Fichte, die der zweiten (rothbraunen) Form auf der Kiefer lebt. Diese Arten nebst Uebergängen werden vorgezeigt.

Was fünftens die hybriden Bildungen anlangt, die aber nicht mehr als eigentliche Varietäten aufgefafst werden können, so scheinen sie bei den Lepidopteren nicht zur bleibenden Varietätenbildung Veranlassung werden zu können, da nach den seitherigen Beobachtungen sich alle Bastarde als unfruchtbar erwiesen haben. Es werden hier die schönen Bastarde von Smerinthus populi L. und ocellata $\mathrm{L}$. vorgezeigt, die Westwood als hybridus beschrieb. Diese ausgezeichnete Mittelform erscheint aber nur dann, wenn Sm. ocellata der Vater und Sm. populi die Mutter ist; umgekehrt erscheint ein Bastard, der durchaus nicht vom Vater (populi) zu unterscheiden ist (wenigstens nach den bisherigen, vielleicht ungenügẹnden Beobachtungen).

Als eine ganz besondere Art der Variation erscheint der Dimorphismus, resp. Polymorphismus, der im Auftreten zweier oder mehrerer ganz verschiedener Formen einer und derselben Art besteht. Derselbe ist ein doppelter und tritt erstens bei den beiden Geschlechtern einer und derselben Art (als sexueller Dimorphismus) auf, wenn dieselben nämlich so verschieden von einander sind, dafs man sie früher (und auch theilweise wohl noch heute) als zwei verschiedene Arten angesehen und beschrieben hat. Zweitens findet er sich nur bei einem Geschlecht, das in zwei oder auch mehr (Polymorphismus) oft ganz verschiedenen Formen auftritt. Eine Combination dieser beiden Arten des Dimorphismus tritt auch gar nicht selten auf, bei welchen einmal die beiden $\mathrm{Ge}$ schlechter von einander verschieden und dann noch das eine $\mathrm{Ge}$ schlecht wieder zwei verschiedene Formen zeigt. So bei den meisten Colias-Arten u. s. w. Aufser dem sehr häufigen sexuellen Dimorphismus sind dem Vortragenden bei den Schmetterlingen nur die Weibchen als dimorph (polimorph) bekannt. Als eins der auffallendsten Beispiele von sexuellem Dimorphismus wird Epicalia nyctimus Westw. aus dem südamerikanischen Faunen - Gebiet vorgezeigt, als auffallende Beispiele des Polymorphismus bei den Weibchen einer Art Papilio Pammon L. und Memnon L. aus dem indomalayischen Faunen - Gebiet, wo von letzteren fünf ganz verschiedene Weibchen nicht nur in Färbung und Zeichnung, sondern theilweise auch in der Form vorgezeigt werden. Auch die früheren Zustände, Raupen und Puppen, zeigen häufig Dimorphismus; 
so werden zwei in Farbe und Zeichnung völlig verschiedene Raupen von Acherontia Atropos L. vorgezeigt.

Schliefslich werden noch einige ausgezeichnete Fälle von Mimicry besprochen und vorgezeigt. Hier sind Arten aus ganz verschiedenen Familien, die normal ein ganz anderes Aussehen haben, einander fast völlig gleich. Am anschaulichsten für Nichtkenner der Lepidopteren-Systematik wird dies durch die Annahme erläutert, dafs z. B. bei den Säugethieren ein Wiederkäuer äus serlich genau einem Raubthiere gleichen würde. Diese zuerst von Bates entdeckte Thatsache kann durch die darwinistische Theorie der natürlichen Züchtung erklärt werden, da die nachgeahmten Schmetterlinge solche sind, welche durch widerlichen Geruch u. s. w. von den Insekten fressenden Vögeln verschont werden sollen. Diesem widersprechend ist freilich die Thatsache, dafs die nachäffenden (mimetischen) Arten meist viel seltener sind, als die anderen Arten derselben Gattung, die normal geblieben sind.

\section{Das traurige Ende des Dr. Fedtschenko,}

über welches wir in dieser Zeitschrift 1873 p. 236 berichtet haben, scheint neueren Mittheilungen zufolge nicht lediglich auf Rechnung der widrigen Naturgewalten zu setzen zu sein. Da die Gefahr, bei Unkenntnifs der Verhältnisse gleichem Geschick zu erliegen, mehr oder weniger jedem Reisenden droht, der im Dienste der Wissenschaft schwierige Erforschungsgebiete zu durchwandern unternimmt, so halten wir es im Interesse der Allgemeinheit für geboten, auch die anklagenden Nachrichten mitzutheilen. Jeder Sachverständige mufste wohl über das a. a. O. auf Seite 237 geschilderte Verhalten der Führer den Kopf schütteln, und kaum konnte der Gedanke unterdrückt werden, dafs Dr. Fedtschenko wohl, wenn nicht fahrlässigen, betrügerischen, so doch mindestens unzuverlässigen, der Situation nicht gewachsenen Führern in die Hände gefallen sei. Diese Befürchtungen haben nun ihre Bestätigung durch eine Genfer Correspondenz der Moskauer Zeitung erhalten, in welcher Herr P. in Chamouny beschuldigt wird, seine Neffen Hrn. Fedtschenko zu Führern empfohlen zu haben, um denselben den Geldgewinn zuzuwenden, obschon er gewufst hat, dafs dieselben einfache Landleute und des Führens ungeübt seien. Diese Führer haben dann in der fahrlässigsten Weise ihre Pflicht vergessen und den Hülfeflehenden aufgegeben und im Stich gelassen. Und in der That, nur in dieser Weise läfst es sich verstehen, dafs der der Reisegefahren kundige und gewohnte Forscher im einfachen Schneesturme elendiglich zu Grunde ging. Allen aber, die von Chamouny aus sicherer Führer bedürfen, wird es nach diesen Mittheilungen gerathen erscheinen, dieselben anderwärts als bei Herrn P. zu suchen.

A. Hensel. 

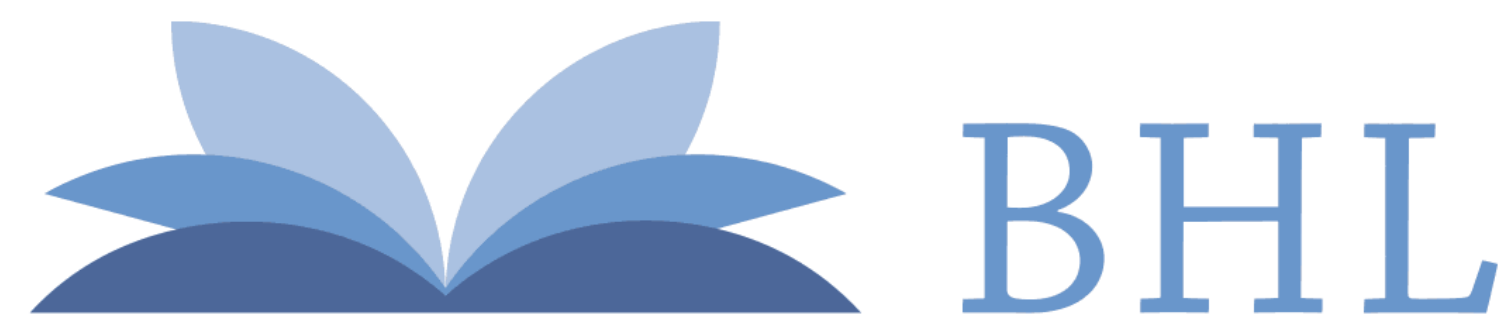

\section{Biodiversity Heritage Library}

1874. "Ueber die Varietätenbildung unter den Schmetterlingen mit Bezug auf die darwinistische Theorie. Vortrag von Dr. Staudinger in der 4ten Sitzung der naturwissenschaftlichen Gesellschaft Isis zu Dresden am 8. Mai 1873. Nach dem Referat in den Sitzungsbericht." Berliner entomologische Zeitschrift / herausgegeben von dem Entomologischen Vereine in Berlin 18(142),147-150. https://doi.org/10.1002/mmnd.18740180131.

View This Item Online: https://www.biodiversitylibrary.org/item/36410

DOI: https://doi.org/10.1002/mmnd.18740180131

Permalink: https://www.biodiversitylibrary.org/partpdf/209995

\section{Holding Institution}

Smithsonian Libraries

\section{Sponsored by}

Smithsonian

\section{Copyright \& Reuse}

Copyright Status: Public domain. The BHL considers that this work is no longer under copyright protection.

This document was created from content at the Biodiversity Heritage Library, the world's largest open access digital library for biodiversity literature and archives. Visit BHL at https://www.biodiversitylibrary.org. 